\title{
The first VLBI image of an infrared-faint radio source
}

\author{
E. Middelberg ${ }^{1}$, R. P. Norris ${ }^{2}$, S. Tingay ${ }^{3}$, M. Y. $\mathrm{Mao}^{4}$, C. J. Phillips ${ }^{2}$, and A. W. Hotan ${ }^{3}$ \\ 1 Astronomisches Institut, Ruhr-Universität Bochum, Universitätsstr. 150, 44801 Bochum, Germany \\ e-mail: middelberg@astro.rub.de \\ 2 Australia Telescope National Facility, PO Box 76, Epping NSW 1710, Australia \\ e-mail: [ray.norris; chris.phillips]@csiro.au \\ 3 Department of Imaging and Applied Physics, Curtin University of Technology, Bentley, Western Australia, Australia \\ e-mail: s.tingay@ivec.org; a.hotan@curtin.edu.au \\ 4 School of Mathematics and Physics, University of Tasmania, Private Bag 21, Hobart TAS 7001, Australia \\ e-mail: minnie.mao@utas.edu.au
}

Received 24 June 2008 / Accepted 8 September 2008

\begin{abstract}
Context. We investigate the joint evolution of active galactic nuclei and star formation in the Universe.

Aims. In the $1.4 \mathrm{GHz}$ survey with the Australia Telescope Compact Array of the Chandra Deep Field South and the European Large Area ISO Survey $-\mathrm{S} 1$ we have identified a class of objects which are strong in the radio but have no detectable infrared and optical counterparts. This class has been called Infrared-Faint Radio Sources, or IFRS. 53 sources out of 2002 have been classified as IFRS. It is not known what these objects are.

Methods. To address the many possible explanations as to what the nature of these objects is we have observed four sources with the Australian Long Baseline Array.

Results. We have detected and imaged one of the four sources observed. Assuming that the source is at a high redshift, we find its properties in agreement with properties of Compact Steep Spectrum sources. However, due to the lack of optical and infrared data the constraints are not particularly strong.
\end{abstract}

Key words. galaxies: active - galaxies: peculiar

\section{Introduction}

Infrared-faint radio sources (IFRS) were recently discovered as a class by Norris et al. (2006), and may be related to the optically invisible radio sources (OIRS) identified by Higdon et al. (2005). IFRS are radio sources which have no counterparts in infrared images from the Spitzer Wide-Area Extragalactic Survey (SWIRE) between $3.6 \mu \mathrm{m}$ and $24 \mu \mathrm{m}$, and are discovered in arcsec-scale radio observations. They are unexpected because it was thought that any galaxy which is detected in radio observations should be detected in the infrared with relatively short integrations. Assuming the SED of known classes of galaxies, a $5 \mathrm{mJy}$ radio source in the local Universe should produce a detectable Spitzer source, regardless of whether it is generated by star formation or AGN (active galactic nuclei). Similarly a normal $L_{*}$ galaxy at $z<1$, whether spiral or elliptical, should be visible in our Spitzer and $I$-band data.

Norris et al. (2006) and Middelberg et al. (2008) together identified 53 such sources out of $2002(2.7 \%)$ detected in the ATLAS survey, co-located with the SWIRE survey. Most of these sources have flux densities of only a few hundred microjansky, but some are strong and have flux densities of more than $20 \mathrm{mJy}$. Stacking $3.6 \mu \mathrm{m}$ Spitzer images at the positions of 22 IFRS, Norris et al. (2006) were unable to make a detection in the averaged image and so demonstrated that IFRS are well below the detection threshold of the SWIRE survey.

The nature of IFRS and the reason for their faintness at infrared wavelengths is unclear. Possible explanations are that (i) these sources are extremely redshifted AGN; (ii) they are dust-rich, extremely obscured galaxies which makes them invisible in the infrared; (iii) they are lobes of nearby, unidentified radio galaxies; or (iv) they are an unknown type of galactic or extragalactic object. Because IFRS have so far only been detected at radio wavelengths it is not possible to measure their redshifts, as spectroscopy requires sub-arcsecond positional accuracy, which the radio observations cannot provide. Also, the comparatively low resolution of the radio images makes it difficult to select the correct optical counterpart, because the corresponding optical observations are deep, and hence confusionlimited.

A promising route to find out more about IFRS is radio observations with very long baseline interferometry (VLBI). VLBI observations are sensitive only to very compact structures with brightness temperatures of the order of $10^{6} \mathrm{~K}$ or more, which are unambiguous signposts of AGN activity. They also yield, when astrometric calibrators are used, positions accurate to milliarcseconds, and milliarcsecond-scale morphologies which can be interpreted in terms of the emission mechanism at work.

Norris et al. (2007) have observed two IFRS with VLBI and detected one. Unfortunately, their $(u, v)$ coverage was too poor to make a reliable image of the detected source. They concluded that the VLBI observations were consistent with a radio-loud AGN at high redshift, or with a lower-power AGN at lower redshift in an abnormally obscured galaxy.

Here we present VLBI observations of four IFRS discovered in the ATLAS/ELAIS field (Middelberg et al. 2008). We selected S427 and S509 because they were the strongest IFRS in 
Table 1. Source parameters and results from our observations.

\begin{tabular}{|c|c|c|c|c|c|c|c|}
\hline Source & IAU designation & RA & Dec & $\begin{array}{c}S_{1.4 \mathrm{GHz}} \\
\mathrm{mJy} \\
(5)\end{array}$ & $\begin{array}{c}S_{\text {VLBI }} \\
\text { mJy } \\
(6)\end{array}$ & $\begin{array}{c}S_{\text {VLBI,max }} \\
\text { mJy } \\
(7)\end{array}$ & $\begin{array}{c}\text { rmsVLBI } \\
\text { mJy } \\
(8)\end{array}$ \\
\hline $0022-423$ & PKS 0022-423 & $00: 24: 42.989741$ & $-42: 02: 03.94796$ & 2820 & $\begin{array}{c}2690 \\
2390^{*}\end{array}$ & $\begin{array}{c}1750 \\
1790^{*}\end{array}$ & $\begin{array}{c}6.4 \\
2.7^{*}\end{array}$ \\
\hline S427 & ATELAIS J003411.59-435817.0 & 00:34:11.59 & $-43: 58: 17.036$ & 21.4 & 12.5 & 8.2 & 0.14 \\
\hline S509 & ATELAIS J003138.63-435220.8 & $00: 31: 38.64$ & $-43: 52: 20.824$ & 22.2 & $\ldots$ & $<0.27$ & 0.065 \\
\hline S433 & ATELAIS J003413.43-435802.4 & $00: 34: 13.43$ & $-43: 58: 02.470$ & 0.2 & $\ldots$ & $<0.27$ & 0.069 \\
\hline S775 & ATELAIS J003216.05-433329.6 & $00: 32: 16.01$ & $-43: 33: 37.092$ & 3.6 & $\ldots$ & $<0.26^{*}$ & $0.055^{*}$ \\
\hline
\end{tabular}

Column 1: source name; Col. 2: formal IAU designation, which for brevity we do not use elsewhere in this paper; Cols. 3, 4: coordinates used in the observations. The target coordinates differ from those published in Middelberg et al. (2008) because they were taken from an early version of the $1.4 \mathrm{GHz}$ ATCA image; Col. 5: ATCA $1.4 \mathrm{GHz}$ flux density by Middelberg et al. (2008) in mJy; Col. 6: integrated flux density found in the VLBI images in mJy; Col. 7: peak flux density of the sources in mJy; Col. 8: rms noise measured from the VLBI images in mJy. The peaks in the last three sources all are below $5 \sigma$, and the locations of the brightest pixel does not coincide with the arcsec-scale source position. The VLBI flux densities were all measured at $1.6 \mathrm{GHz}$, with the exception of those marked with a superscript asterisk, which were made at $1.4 \mathrm{GHz}$.

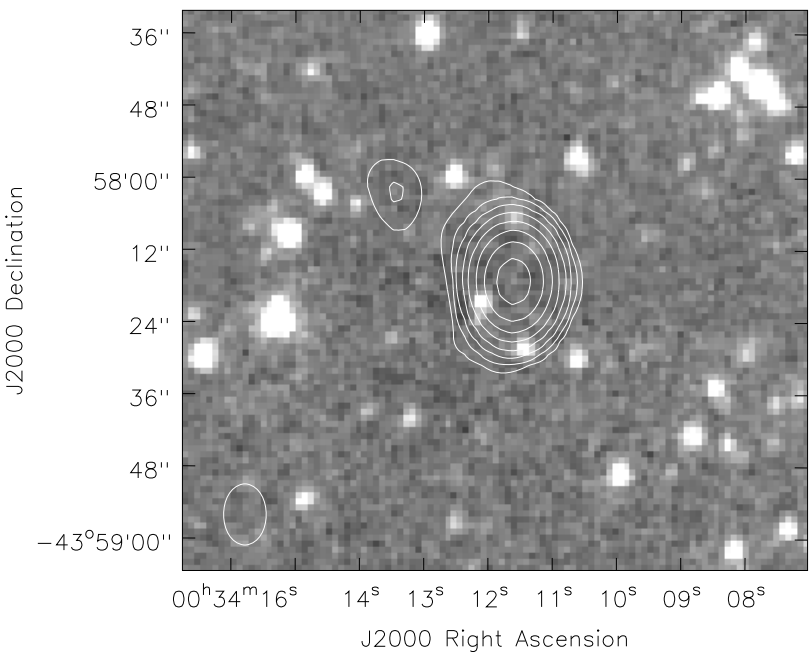

Fig. 1. Contour plot of the ATCA $20 \mathrm{~cm}$ image of $\mathrm{S} 427$ superimposed on the $3.6 \mu \mathrm{m}$ Spitzer image made as part of the SWIRE survey (Lonsdale et al. 2003). Contours are drawn at $0.1 \mathrm{mJy} \times(1,2,4, \ldots)$ and the restoring beam was $10.3 \times 7.2$ arcsec. The nearest infrared source, located towards the south-east, is more than 6 arcsec away, making it very unlikely to be the infrared counterpart. Source S433, visible as a twocontour object 24.6 arcsec north-east of S427, also was classified as an IFRS. It has a flux density of $245 \mu \mathrm{Jy}$.

the ATLAS/ELAIS field, and S775 because it is very extended on arcsecond scales, showing structures reminiscent of lobes and jets frequently seen in AGN. After the observations it was discovered that the weak IFRS S433 was located only 24.6 arcsec north-east of S427 (Fig. 1), and was well within the field of view of the VLBI array. The details of the sources observed are listed in Table 1.

\section{Observations}

We observed the IFRS with the Australian Long Baseline Array (LBA) in phase-referencing mode. On 24 March 2007 the observing frequency was $1.6 \mathrm{GHz}$ and the participating antennas were the Australia Telescope Compact Array (ATCA), the $64 \mathrm{~m}$ Parkes telescope, the $22 \mathrm{~m}$ Mopra telescope and the $26 \mathrm{~m}$ telescope near Hobart. On 21 June 2007 the observing frequency was $1.4 \mathrm{GHz}$ and the same array was used, plus the $30 \mathrm{~m}$ telescope at Ceduna. The ATCA, Parkes and Mopra telescopes recorded a total bandwidth of $64 \mathrm{MHz}$ in both
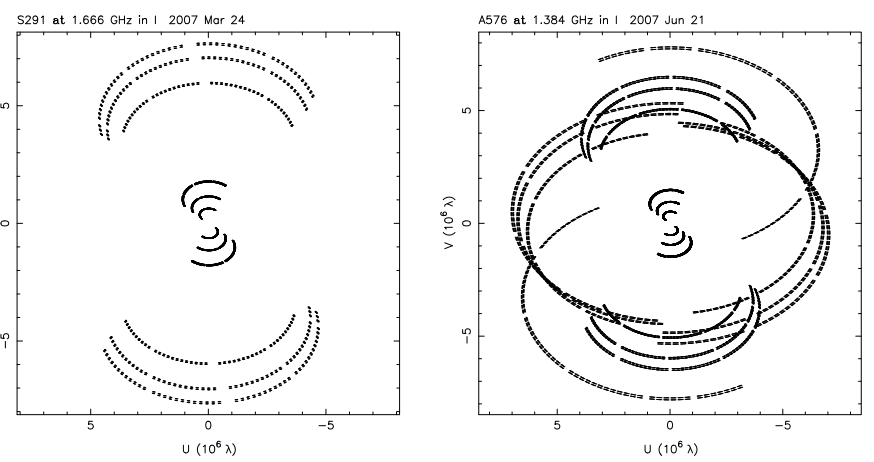

Fig. 2. Plots showing the $(u, v)$ coverage of the observations of S427 on 24 March (left) and of S775 on 21 June (right).

polarizations, using 2-bit sampling, subdivided in $16 \mathrm{MHz}$ wide IF channels. Both right-hand and left-hand circular polarization were recorded. The Hobart and Ceduna telescopes recorded a total bandwidth of $32 \mathrm{MHz}$ with the same setup. Both observing runs lasted for $12 \mathrm{~h}$. The total $(u, v)$ coverage of the two observing runs is shown in Fig. 2.

These are the first published LBA observations using Ceduna at $1.4 \mathrm{GHz}$. The design of Ceduna (an old telecommunications dish donated to the University of Tasmania in 1995) had not foreseen observations below several $\mathrm{GHz}$, and frequencies lower than $2 \mathrm{GHz}$ are blocked by waveguides in the Nasmyth focus. Efforts are now underway to work around this limitation by installing a tertiary mirror at the centre of the main reflector. This bypasses the Nasmyth focus entirely and allows the reception of frequencies below $2 \mathrm{GHz}$. At the time of our observations, a test system consisting of a fixed tertiary and an uncooled $1.4 \mathrm{GHz}$ receiver had been installed, yielding a system temperature of around $1260 \mathrm{~K}$ in RCP and $1390 \mathrm{~K}$ in LCP. In the near future, a cooled receiver and moveable tertiary reflector will allow Ceduna to routinely participate in VLBI observations at sky frequencies between $1.2 \mathrm{GHz}$ and $1.8 \mathrm{GHz}$, in addition to its existing higher frequency capabilities.

On 24 March, the coordinates of IFRS S427 and S509 were observed for $5 \mathrm{~min}$ each, followed by a $3 \mathrm{~min}$-scan on the nearby phase calibrator 0022-423, which has an arcsec-scale flux density (monitored with the ATCA) of $2.82 \mathrm{Jy}$. S433 was also in the field of view of these observations. On 21 June, 8 min-scans of the IFRS S775 were followed by a 3 min-scan of the same calibrator. One of the fringe finders 1921-293 and 0104-408 was observed every two hours in either run. The LBA sensitivity 

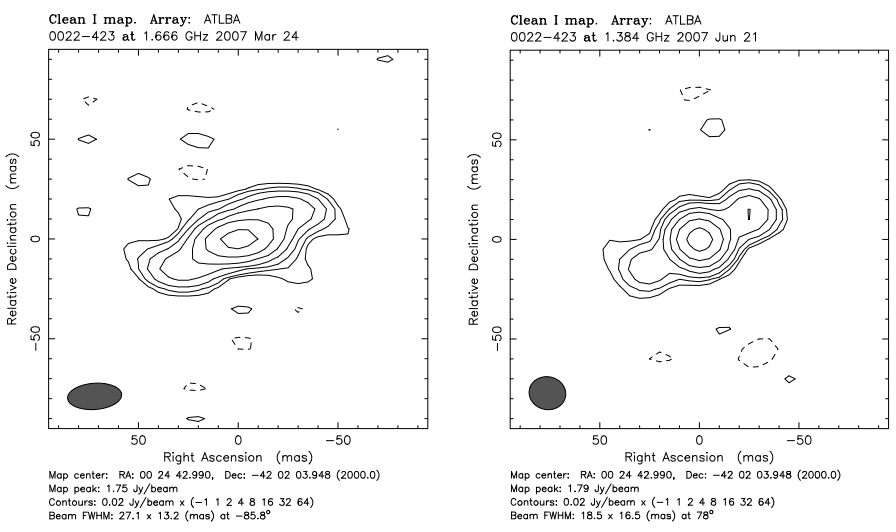

Fig. 3. Contour plots of the phase calibrator 0022-423 observed on 24 March (left) and 21 June (right). Contours are drawn at $20 \mathrm{mJy} \times(-1$, $1,2,4, \ldots)$ and the restoring beam was 27.1 mas $\times 13.2$ mas $(l e f t)$ and 18.5 mas $\times 16.5$ mas (right). The observations on 21 June used a slightly improved position for the calibrator.

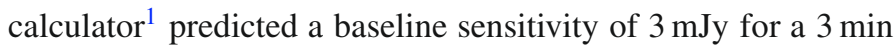
calibrator scan on the least sensitive baseline between Mopra and Hobart. The predicted image sensitivities, using all data, were around $50 \mu \mathrm{Jy}$ for the targets.

\section{Calibration}

The lower and upper 10 out of 64 channels in each IF had low amplitudes and were flagged. Also, after source changes the correlator produced data although the antennas had not yet arrived at the source positions, requiring the flagging of $30 \mathrm{~s}$ of the beginning of all scans, and occasionally more. A short section of a fringe-finder observation was fringe-fitted to obtain residual and instrumental delays, and phase offsets for each IF and polarization separately. These solutions were applied to the entire data set, and allowed averaging across the band in the subsequent fringe-fitting of the phase calibrator. The phase calibrator was detected on all baselines throughout the experiments with a high signal-to-noise ratio (SNR). Initial amplitude calibration was carried out using $T_{\text {sys }}$ values measured during the observations, and known antenna gains. The phase calibrator was then imaged in Difmap (Shepherd 1997). Amplitude self-calibration was performed in Difmap with a 720 min solution interval, yielding small $(<20 \%)$ corrections for the antenna gains. The complex gains from this procedure were then applied to the targets. Contour plots of the calibrator are shown in Fig. 3. An area of $2^{\prime \prime}$ centred on the targets' arcsec-scale emission was imaged to look for the sources.

\section{Results and discussion}

Only one of the three targets, S427, was detected with good SNR. The other three fields were completely devoid of emission. In the case of S433 the loss of sensitivity due to wide-field effects (bandwidth smearing, time smearing, and primary beam attenuation) is of the order of only a few percent on the longest baselines, and hence is negligible. Therefore its non-detection is just as significant as the other non-detections.

${ }^{1}$ http://www.atnf.csiro.au/vlbi/calculator

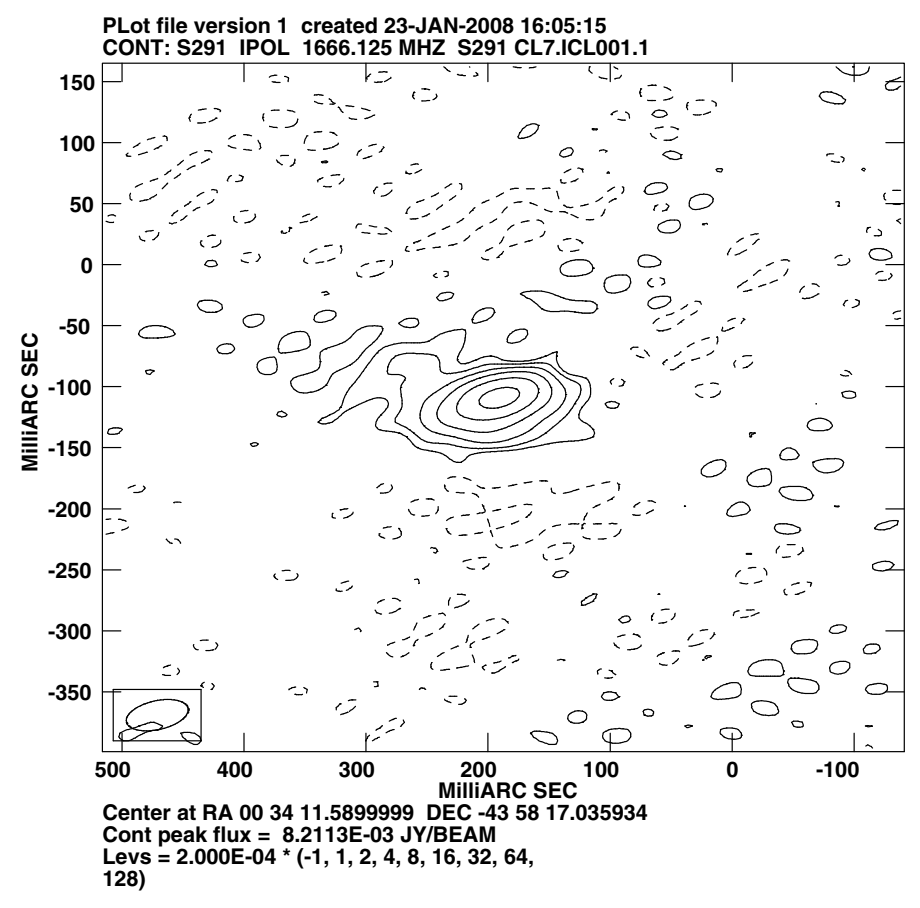

Fig. 4. Contour plot of the IFRS S427. Contours are drawn at $0.2 \mathrm{mJy} \times(-1,1,2,4, \ldots)$ and the restoring beam was 51.7 mas $\times 23.6$ mas.

\section{1. $S 427$}

The image of S427 in Fig. 4 displays a slightly, though significantly extended point source, with the highest sidelobes at a level of $0.3 \mathrm{mJy}$. Even though the extension is weak, it is unlikely to be an imaging artefact because a) the image quality could be improved considerably by including the extension in the model, and b) because the flux density measured on the shortest baseline was about a factor of two higher than that measured on the longest baseline. The peak flux density in the image is $8.2 \mathrm{mJy}$ and the integrated flux density is $12.5 \mathrm{mJy}$. The limited $(u, v)$ coverage defied attempts to develop a more detailed model, and caused the noise in regions away from the source to be dominated by sidelobes rather than receiver noise. On the longest baselines the source has a flux density of $7 \mathrm{mJy}$, from which we infer a minimum brightness temperature of $T_{\mathrm{B}, \min }=3.6 \times 10^{6} \mathrm{~K}$, indicating non-thermal emission. We therefore conclude that the source contains an AGN.

A VLBI image is a significant step towards understanding these mysterious objects. First, we now know that the VLBI detection by Norris et al. (2007) is not a single, isolated event. Although VLBI detections appear not to be the rule, they also do not appear to be rare. Second, the extension visible in Fig. 4 allows one to make statements about the source geometry and to compare it to observations at lower resolution.

S427 has an arcsec-scale flux density of $(21.4 \pm 1.07) \mathrm{mJy}$ (Middelberg et al. 2008), 58\% of which we recovered on baselines longer than $500 \mathrm{k} \lambda$, or on scales smaller than 410 mas, and $33 \%$ of which are detected on baselines longer than $6.5 \mathrm{M} \lambda$, or on scales smaller than 32 mas.

\subsection{The spectrum of $S 427$}

Following up on these results, on 27 and 29 April 2008 we observed S427 with the ATCA at $4.8 \mathrm{GHz}$ and $8.6 \mathrm{GHz}$, using director's time during a maintenance period. At $4.8 \mathrm{GHz}$ we 
were able to combine data from the $6 \mathrm{~A}$ and $750 \mathrm{~A}$ configurations whereas the $8.6 \mathrm{GHz}$ data were only observed in the $750 \mathrm{~A}$ configuration, and therefore yielded an image with a lower resolution and image fidelity than the $4.8 \mathrm{GHz}$ data. The bandwidth was $128 \mathrm{MHz}$ in two polarizations, and the integration times were $11 \mathrm{~h}$ at $4.8 \mathrm{GHz}$ and $5.6 \mathrm{~h}$ at $8.6 \mathrm{GHz}$.

The $4.8 \mathrm{GHz}$ image (not shown) displays a marginally resolved source which is adequately described by a Gaussian with $B_{\max }=5.7^{\prime \prime}$ and $B_{\min }=3.5^{\prime \prime}$ in PA $=-39^{\circ}$ (north through east). The deconvolved size was found to be $1.6^{\prime \prime} \times 0.7^{\prime \prime}$ in $\mathrm{PA}=-63^{\circ}$, so the source is close to being unresolved. The $8.4 \mathrm{GHz}$ image (also not shown) displays a slightly extended source with $4.2^{\prime \prime} \times 3.6^{\prime \prime}$ in $\mathrm{PA}=51^{\circ}$ and a deconvolved size of $2.8^{\prime \prime} \times 2.5^{\prime \prime}$ in PA $=58^{\circ}$. The integrated flux densities were found to be $S_{4800}=(6.4 \pm 1.3) \mathrm{mJy}$ and $S_{8640}=(1.8 \pm 0.4) \mathrm{mJy}$.

The PA of the deconvolved Gaussian at $8.6 \mathrm{GHz}$ is in approximate agreement with the PA found at $1.4 \mathrm{GHz}$, but this extension has not been found at $4.8 \mathrm{GHz}$. Our observations at $8.4 \mathrm{GHz}$ had significantly lower SNR than those at $4.8 \mathrm{GHz}$, which may have affected the deconvolution. However, the SNR at $8.4 \mathrm{GHz}$ is too high to be the only reason for this discrepancy, and therefore the deconvolved size of S427 at this frequency is not understood.

From a $2.3 \mathrm{GHz}$ follow-up survey of the ATLAS/ELAIS field currently being conducted by us with the ATCA, and from the SUMSS survey (Bock et al. 1999) we have obtained two more spectral points for S427. The catalogued flux density from the SUMSS survey at a frequency of $843 \mathrm{MHz}$ and with a resolution of $45^{\prime \prime} \times 64^{\prime \prime}$ was $(42.7 \pm 1.5) \mathrm{mJy}$, and from our preliminary $2.3 \mathrm{GHz}$ image at a centre frequency of $2.424 \mathrm{GHz}$ and with resolution of $52^{\prime \prime} \times 28^{\prime \prime}$ we have obtained a flux density of (12.5 \pm 1.9$) \mathrm{mJy}$. We have convolved the $1.4 \mathrm{GHz}$ ATLAS/ELAIS image (with a centre frequency $1.382 \mathrm{GHz}$ and a resolution of $10.3^{\prime \prime} \times 7.2^{\prime \prime}$, but sufficient short-baseline coverage for a lowresolution image) with an appropriate Gaussian kernel to generate an image with a resolution matching that at $2.3 \mathrm{GHz}$, and found a flux density of $22.7 \mathrm{mJy}$. This is within the errors of the flux density measured from the higher-resolution image and indicates that there is no faint emission on scales of tens of arcsec, as would be expected from a very compact source. The spectral indices therefore can be computed from the full-resolution $1.4 \mathrm{GHz}$ image, and are $\alpha_{1.382}^{843}=-1.39$ and $\alpha_{2.424}^{1.382}=-0.96$.

The five flux density measurements now available are shown in Fig. 5. We also show a power-law function fitted to the data, which is able to represent the data rather well, and which has an exponent of -1.31 , showing that the source has a very steep spectrum over a decade in frequency.

\subsection{Modelling the properties of $S 427$ at two redshifts}

Following the discussion by Norris et al. (2007), we model the emission of S427 assuming it has redshifts of $z=1$ and $z=7$ (the results of which we give in brackets). The measured flux densities need to be corrected for redshift ( $k$-correction) and spectral index $\left(\alpha, S \propto v^{\alpha}\right)$. S427 has a $4.8 \mathrm{GHz}$ flux density of $6.4 \mathrm{mJy}$ which, when corrected for redshift and spectral index, corresponds to a rest-frame $5 \mathrm{GHz}$ luminosity of $7.9 \times 10^{25} \mathrm{~W} \mathrm{~Hz}^{-1}\left(5.5 \times 10^{28} \mathrm{~W} \mathrm{~Hz}^{-1}\right.$ if $z=7$, using $H_{0}=71$, $\Omega_{\mathrm{M}}=0.27$ and $\left.\Omega_{\mathrm{vac}}=0.73\right)$. At $z=7$ this is approximately the luminosity of 3C 273, hence is high (e.g., O'Dowd et al. 2002), but not impossible.

The size of the source can be estimated as follows. In the $1.4 \mathrm{GHz}$ ATCA image the source was well represented with a Gaussian of $10.62^{\prime \prime} \times 7.89^{\prime \prime}$ in PA $174^{\circ}$. After deconvolution from the restoring beam, the intrinsic source size was found

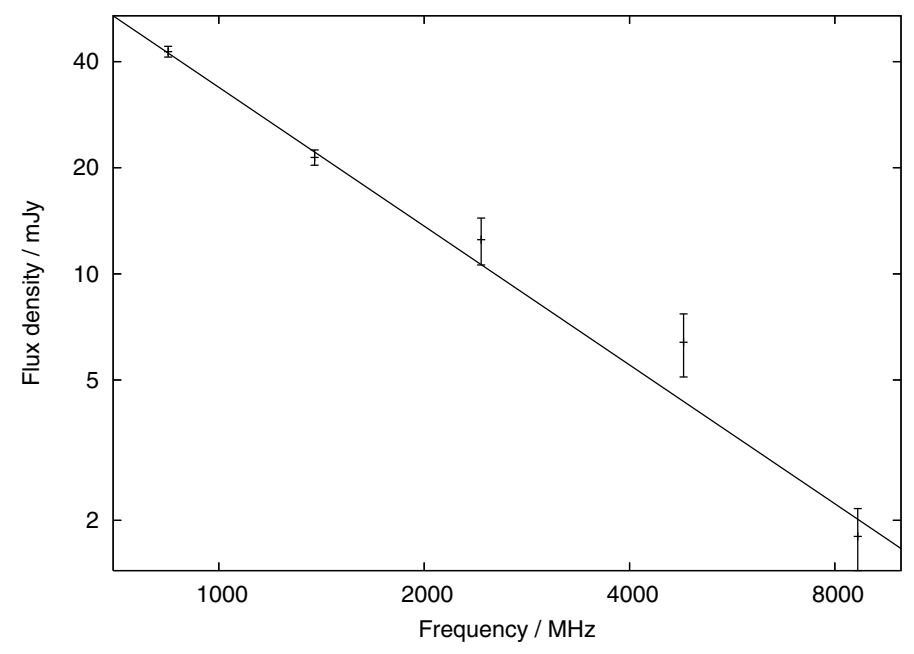

Fig. 5. The arcsec-scale radio spectrum of $\mathrm{S} 427$ between $843 \mathrm{MHz}$ and $8640 \mathrm{MHz}$, and a power-law fitted to the data.

to be $3.30^{\prime \prime} \times 2.74^{\prime \prime}$ in PA $95^{\circ}$. At $z=1$ the linear scale is $8.0 \mathrm{kpcarcsec}^{-1}\left(5.3 \mathrm{kpc} \operatorname{arcsec}^{-1}\right)$, and so the intrinsic source size is of the order of $24 \mathrm{kpc}(16 \mathrm{kpc})$.

We point out that the position angles of deconvolved sources in the source catalogue by Middelberg et al. (2008) are very evenly distributed and do not appear to be biased in any direction. Hence the orientation of S427, when deconvolved from the restoring beam in the arcsec-scale image, is in agreement with the extension seen in the VLBI image.

It is surprising that $\mathrm{S} 509$ has spectral properties which are very similar to S427, with $S_{2.424}=12.6 \mathrm{mJy}, S_{843}=37.6 \mathrm{mJy}$, and thus $\alpha_{1.382}^{843}=-1.06$ and $\alpha_{2.424}^{1.382}=-1.01$. Its intrinsic size at $1.4 \mathrm{GHz}$ after deconvolution is $3.48^{\prime \prime} \times 2.77^{\prime \prime}$ in PA $59^{\circ}$. Yet it remains undetected in our VLBI observations which indicates that if it contains an AGN it is comparatively weak or currently inactive.

\subsection{The nature of $S 427$}

Given its brightness temperature, S427 must contain an AGN, and this AGN accounts for $58 \%$ of its total flux density. Given its compactness, it is not a radio lobe of a yet unidentified radio galaxy, which was one of the possible explanations. Also, its steep spectrum indicates that the radio emission from S427 is not dominated by star formation, which is expected to produce a spectral index of around -0.7 .

S427 does not appear to be a "standard" FR I/II radio galaxy. Its linear size of less than $24 \mathrm{kpc}$ if between redshifts of 1 and 7 , derived from the ATCA observations, is smaller by a factor of a few than what is typically observed in FR I galaxies (although very few are quite smaller than that), and its $1.4 \mathrm{GHz}$ luminosity of $\log (L)=26.05 \mathrm{~W} \mathrm{~Hz}^{-1}$ if $z=1$ and $\log (L)=28.11 \mathrm{~W} \mathrm{~Hz}^{-1}$ if $z=7$ is at the very high end of what is being observed (Owen \& Ledlow 1994). FR II radio galaxies are even larger than FR Is, but their luminosities can be as high as that of S427 if at $z=7$. Furthermore, in FR I/II radio galaxies almost all of the emission comes from the extended radio lobes, in particular at low frequencies (due to the steep spectral index of the radio lobes). In $\mathrm{S} 42733 \%$ of the emission comes from scales smaller than $250 \mathrm{pc}$, which would be unusual for an FR II. We conclude that although we cannot rule out that $\mathrm{S} 427$ is a FR I/II radio galaxy, its extent, luminosity and internal distribution of emission make it unlikely. 
A large fraction ( 40\%, O'Dea 1998) of radio sources are the compact steep-spectrum (CSS) and gigaherz-peaked spectrum (GPS) sources. CSS and GPS sources are very small yet strong radio sources. They are contained within their host galaxies, with CSS sources being smaller than $20 \mathrm{kpc}$, whereas GPS sources are smaller than $1 \mathrm{kpc}$, so that they are smaller than the narrow-line region. The two competing models to explain their properties are that they are either "frustrated" radio galaxies which are confined by their host galaxy's very dense ISM, or that they are young objects which eventually will evolve into large radio galaxies. A detailed review can be found in O'Dea (1998). Given its extent of more than $1 \mathrm{kpc}$ and its power-law spectrum between $843 \mathrm{MHz}$ and $8640 \mathrm{MHz}$, we can rule out that S427 is a GPS source.

A general requirement for sources classified as CSS is a spectral index of less than -0.5 , a source size of less than about $20 \mathrm{kpc}$, and $1.4 \mathrm{GHz}$ luminosities of more than $10^{25} \mathrm{~W} \mathrm{~m}^{-2} \mathrm{~Hz}^{-1}$ (O'Dea 1998). S427 has $\log \left(L_{1.4}\right)=26.1$ if $z=1$ and $\log \left(L_{1.4}\right)=$ 28.1 if $z=7$. Its size and luminosity are therefore in good agreement with typical CSS luminosities, if S427 is at high redshifts.

Using VLBI observations of seven strong CSS, Tzioumis et al. (2002) detect double-lobed structures in all objects. They also find that in most of their objects more than $50 \%$ of the arcsec-scale flux density is contained in the VLBI images. However, in one case the amount of emission resolved out by the VLBI observations is as high as $70 \%$. Within the limits of our observations, S427 does not display a double-lobed structure on mas scales and so is not a typical CSS.

The infrared properties of CSS sources are not well studied, so it is difficult to make a statement about whether S427's IR properties are typical for a CSS or not. Heckman et al. (1994) find that the ratio of IR to radio luminosity of radio galaxies is relatively independent of the object class (FR II, quasar, GPS/CSS, and so on). This was confirmed in similar studies carried out by Hes et al. (1995), and by Fanti et al. (2000). However, these authors all studied predominantly $3 \mathrm{C}$ sources which are selected to be very bright in the radio, and so their samples are plagued by Malmquist bias and their conclusions may not be applicable to IFRS. However, given the lack of other data we estimate the IR luminosity of a CSS at high redshift. Heckman et al. (1994) present a sample of IRAS-detected GPS/CSS sources. Their $12 \mu \mathrm{m}$ flux densities typically are of the order of tens of mJy, but only one object classified as CSS by O'Dea (1998), 3C 48, has been detected at wavelengths between $12 \mu \mathrm{m}$ and $100 \mu \mathrm{m}$. 3C 48 has a redshift of 0.367 , and if shifted to $z=1$ would have a (k-corrected) $24 \mu \mathrm{m}$ flux density of $S_{24 \mu \mathrm{m}}=$ $10.6 \mathrm{mJy}$ and a $3.6 \mu \mathrm{m}$ flux density of $S_{3.6 \mu \mathrm{m}}=0.5 \mathrm{mJy}$, which would have been detected in the SWIRE survey. However, at $z=7$ it would appear as a source with $S_{24 \mu \mathrm{m}}=4.6 \mu \mathrm{Jy}$ and $S_{3.6 \mu \mathrm{m}}=0.9 \mu \mathrm{Jy}$, which is much too faint for the SWIRE survey. Hence if S427's host galaxy is similar to that of 3C 48 it must be at high redshift.

Dicken et al. (2008) have recently carried out an investigation of the radio and infrared properties of powerful, radio-loud radio galaxies at intermediate redshifts using literature data,
ATCA, VLA and Spitzer observations. Their data suggest that the $24 \mu \mathrm{m}$ flux densities of radio galaxies are typically one to two orders of magnitude lower than the $1.4 \mathrm{GHz}$ flux densities, independent of object type. Their study includes the three known CSS sources PKS 0252-71, PKS 1151-34, PKS 1814-63 and the GPS source PKS 1934-63. The radio and infrared properties of these sources follow the trend of their sample. For S427 that implies that the expected $24 \mu \mathrm{m}$ flux density would be between $0.2 \mathrm{mJy}$ and $2 \mathrm{mJy}$ (compared to $160 \mathrm{mJy}$ Heckman et al. 1994, find for 3C 48). The $1 \sigma$ sensitivity of the $24 \mu \mathrm{m}$ ATLAS/Spitzer observations is $252 \mu \mathrm{Jy}$ hence the non-detection of $24 \mu \mathrm{m}$ emission in the ATLAS survey is consistent with the observations by Dicken et al. (2008). However, we note that their work is concerned with very bright radio sources, and that the conclusions made are unlikely to be applicable to a poorly understood class of objects such as the IFRS.

\section{Conclusions}

We present the first VLBI image of an Infrared-Faint Radio Source and report the non-detection of three more IFRS discovered in the ATCA $1.4 \mathrm{GHz}$ observations of the ATLAS/ELAIS field. The main result of our observations is that S427 harbours an AGN, and that it is not simply a radio lobe of an unidentified radio galaxy. The size, spectrum, and radio and IR luminosity of the detected IFRS S427 are consistent with those of a highredshift Compact Steep-Spectrum source, and are inconsistent with a standard $L_{*}$ galaxy at $z<1$, or a FR I/II galaxy at any redshift.

Together with the two IFRS observed by Norris et al. (2007) the number of IFRS observed with VLBI is now 6 , and the number of detections is 2, showing that at least a fraction of IFRS is associated with AGN. We plan further VLBI studies to determine the nature of these enigmatic objects.

Acknowledgements. We thank the staff of the Australian Long Baseline Array for making the observations presented in this paper possible, and we thank Brett Reid for installing the $1.4 \mathrm{GHz}$ test feed on the Ceduna antenna.

\section{References}

Bock, D. C.-J., Large, M. I., \& Sadler, E. M. 1999, AJ, 117, 1578 Dicken, D., Tadhunter, C., Morganti, R., et al. 2008, ArXiv e-prints, 803 Fanti, C., Pozzi, F., Fanti, R., et al. 2000, A\&A, 358, 499

Heckman, T. M., O’Dea, C. P., Baum, S. A., \& Laurikainen, E. 1994, ApJ, 428, 65

Hes, R., Barthel, P. D., \& Hoekstra, H. 1995, A\&A, 303, 8

Higdon, J. L., Higdon, S. J. U., Weedman, D. W., et al. 2005, ApJ, 626, 58 Lonsdale, C. J., Smith, H. E., Rowan-Robinson, M., et al. 2003, PASP, 115, 897 Middelberg, E., Norris, R. P., Cornwell, T. J., et al. 2008, AJ, 135, 1276

Norris, R. P., Afonso, J., Appleton, P. N., et al. 2006, AJ, 132, 2409

Norris, R. P., Tingay, S. J., Phillips, C., et al. 2007, MNRAS

O'Dea, C. P. 1998, PASP, 110, 493

O'Dowd, M., Urry, C. M., \& Scarpa, R. 2002, ApJ, 580, 96

Owen, F. N., \& Ledlow, M. J. 1994, 54, 319

Shepherd, M. C. 1997, in Astronomical Data Analysis Software and Systems VI, ASP Conf. Ser., 125, 77

Tzioumis, A., King, E., Morganti, R., et al. 2002, A\&A, 392, 841 